\title{
La Oficina de Cooperación en Investigación y Desarrollo Tecnológico de la Universitat Jaume I como herramienta de apoyo a la gestión de la I+D
}

\author{
I. RODRIGO, C. VILLARROYA, P. SINISTERRA \\ UJI. Castellón
}

\begin{abstract}
La Oficina de Cooperación en Investigación y Desarrollo Tecnológico (OCIT) de la Universitat Jaume I es la unidad encargada de la gestión de la investigación, la innovación tecnológica y la transferencia de tecnología en su seno. La OCIT es el resultado de la fusión en 1996 entre las extintas Secretaría Técnica de Investigación, servicio que gestionaba la investigación académica, y la Oficina de Transferencia de Resultados de Investigación. La fusión y consecuente creación de la OCIT persiguió objetivos de racionalidad, eficacia y profesionalización en un área que hasta el momento era gestionada por académicos y no por técnicos especialistas. Al mismo tiempo se buscaba un modelo integral de gestión de la I+D que aprovechara los recursos de gestión e información para la mejora de la actividad investigadora, ya fuera ésta de carácter académico o bajo contrato. La OCIT desarrolla una gestión integral de los procesos de apoyo a la investigación: Contratación de I+D vía artículo 11 de la LRU; Participación en Programas de I+D; Difusión y transferencia de tecnologí; y Evaluación de la Investigación. De cada uno de ellos se exponen las estrategias aplicadas, su resultado e impacto con especial referencia a la I+D universitaria en tecnologías cerámicas. (Datos correspondientes al año 1999)
\end{abstract}

The office for cooperation in research and technologic development as a suport tool to management of $R+D$

\section{INTRODUCCIÓN}

Las instituciones de enseñanza superior, llamadas tradicionalmente a ejercer la doble función de docencia e investigación, se han visto requeridas en los últimos años a implicarse de forma activa en el desarrollo económico, social y cultural de su entorno. En lo que respecta a la investigación y desarrollo tecnológico esta implicación requiere dos enfoques:

i) Por una parte es necesario mantener la libertad de investigación. Cabe no perder de vista que la universidad es el único ámbito en donde la ciencia no se desarrolla bajo un enfoque utilitarista, sino todo lo contrario: el investigador universitario puede dejarse guiar por el único acicate de desvelar nuevos conocimientos, y en esta actitud está el fundamento del avance científico y, consecuentemente, del desarrollo técnico.

ii) Por otra parte es bien cierto que la universidad puede y debe contribuir a resolver problemas concretos (técnicos, sociales, medioambientales...) mediante la investigación y el desarrollo tecnológico. Para ello dispone de los recursos humanos y materiales necesarios y, además, el mandato legal que emana de la Ley 11/1983 de Reforma Universitaria en su artículo...

La necesidad y la exigencia social de que la universidad esté en frentes tan distintos y, en muchas ocasiones, antagónicos, propició en los años 80 un debate encendido entre los partidarios de la universidad "academia" y los partidarios de la universidad "empresa", que actualmente se ha diluido en una mero reto organizativo que haga posible la coexistencia e incluso la sinergia entre las actividades de investigación académica y la investigación y los servicios tecnológicos bajo contrato.

El establecimiento de un sistema de gestión de la I+D ágil e integrado en la dinámica universitaria no es algo sencillo. Las estructuras creadas al efecto deben relacionarse de manera fluida con el interior y el exterior de la universidad, deben acumular información acerca de las capacidades internas y las demandas externas y deben dotarse de profesionales preparados en diversas disciplinas; desde el conocimiento técnico básico de las distintas capacidades investigadoras hasta aspectos legales y de gestión.

La Oficina de Cooperación en Investigación y Desarrollo Tecnológico (OCIT) de la Universitat Jaume I es la unidad encargada de la gestión de la investigación, la innovación tecnológica y la transferencia de tecnología en su seno. A continuación se describen algunos aspectos relacionados con su creación, sus objetivos y funcionamiento, así como de sus resultados y su impacto.

\section{MARCO INSTITUCIONAL}

La Universitat Jaume I es una universidad joven (10 años) que cuenta en la actualidad con 818 profesores y 12650 estudiantes. Pese a su tamaño reducido posee una gran variedad de estudios, estructurados en tres centros: la Facultad de Ciencias Jurídicas y Económicas, la Facultad de Ciencias Humanas y Sociales y la Escuela Superior de Tecnología y Ciencias Experimentales.

La función investigadora se organiza en 19 departamentos y 3 institutos universitarios. Actualmente se encuentran censados 118 grupos de investigación. 
El gasto en I+D supone el $31 \%$ de su presupuesto global ${ }^{1}$. Las principal áreas científicas en gasto de I+D son informática/matemáticas e ingeniería (incluida ingeniería química), ambas con el 22\% sobre el total, seguidas de las áreas de economía y dirección de empresas (11\%) y química $(9 \%)$.

La Universitat Jaume I está situada en un área de un marcado monocultivo industrial: el llamado clúster cerámico de Castellón que concentra más del 90\% de la producción española en baldosas cerámicas. Este hecho ha marcado la orientación de sus estudios y de su investigación, que de forma natural han ido adaptándose a la demanda de las empresas del sector.

\section{ANTECEDENTES}

La Universitat Jaume I se ha dotado dos estructuras de interfaz, que canalizan las relaciones de los investigadores con las empresas del entorno, y específicamente, aquéllas que se producen con el sector cerámico. Estas dos estructuras son la Fundación Universitat Jaume I - Empresa (FUJIE) y la Oficina de Cooperación en Investigación y Desarrollo Tecnológico (OCIT).

La OCIT es el resultado de la fusión en 1996 entre las extintas Secretaría Técnica de Investigación, servicio que gestionaba la investigación académica, y la Oficina de Transferencia de Resultados de Investigación. La fusión y consecuente creación de la OCIT persiguió objetivos de racionalidad, eficacia y profesionalización en un área que hasta el momento era gestionada por académicos y no por técnicos especialistas. Al mismo tiempo se buscaba un modelo integral de gestión de la I+D que aprovechara los recursos de gestión e información para la mejora de la actividad investigadora, ya fuera ésta de carácter académico o bajo contrato.

El hecho de que una universidad de pequeño tamaño haya puesto en funcionamiento diversas estructuras de interfaz que, desde ópticas diferentes y con cometidos complementarios, abordan la promoción de la relación universidad-empresa, da cuenta de que la Universitat Jaume I está impregnada de una cultura de cooperación tecnológica y de servicio a su entorno.

En cuanto a la dedicación a la tecnología cerámica la Universitat Jaume I desde su creación ha desarrollado una intensa y creciente relación con el entorno industrial perteneciente a este sector. Ya desde la constitución del Colegio Universitario de Castellón, dependiente de la Universitat de València - Estudi General, un grupo de profesores que trabajaba en tecnología cerámica se fue ubicando progresivamente en Castellón. Con ello se daba respuesta a la creciente demanda de apoyo técnico e I+D solicitada por las empresas de pavimentos y revestimientos cerámicos y de fritas y esmaltes. Este grupo de profesores se organizó como Unidad Predepartamental de Ingeniería Química, desarrollando su actividad investigadora en el Instituto Universitario de Tecnología Cerámica*.

Con la creación de la nueva Universidad en 1991 se produce la incorporación de nuevos investigadores y una reorganización departamental. La unidad predepartamental de Química Inorgánica y Orgánica se vuelca en la investigación y desarrollo de fritas y esmaltes, mientras que otros departamentos han iniciado líneas de investigación relacionadas con la industria cerámica, confirmando la atracción que suscita la problemática tecnológica de este sector, uno de los más activos de la Comunidad Valenciana.

\section{MISIÓN}

La OCIT es la unidad encargada de promover y gestionar las actividades de investigación y de innovación tecnológica de la Universidad Jaume I en dos vertientes

El reforzamiento de la conexión entre la capacidad generadora de conocimientos científicos y tecnológicos de la Universidad y las demandas de los sectores industriales, que posibilite una plena integración de los investigadores universitarios en el sistema de innovación industrial valenciano.

La dinamización de la actividad investigadora de la Universitat Jaume I, estimulando y facilitando la presencia sus grupos de $\mathrm{I}+\mathrm{D}$ en los programas valencianos, españoles o europeos de investigación y desarrollo tecnológico.

\section{5. ÁREAS DE TRABAJO Y OBJETIVOS PARTICULARES}

La OCIT desarrolla una gestión integral de los procesos de apoyo a la investigación, a excepción de la gestión de las infraestructuras de apoyo que están confiadas al Servicio de Informática en lo que respecta al cálculo científico y a los Servicios Centrales de Instrumentación Científica en lo que respecta a los grandes equipos. Las tareas de documentación científica, confiadas a la Biblioteca, también quedan fuera del ámbito de gestión de la OCIT.

Así pues, la OCIT actúa en las siguientes áreas para obtener los objetivos generales que se señalan para cada una de ellas:

i) Contratación de I+D vía artículo 11 de la LRU: La contratación de I+D supone la materialización de uno de los objetivos de la Universidad: contribuir con sus medios materiales y humanos al desarrollo industrial, y por ende socioeconómico, de su entorno. Los investigadores de la Universidad pretenden, a través de este proceso, diversificar sus fuentes de financiación y conectar su actividad investigadora con las necesidades industriales. Las empresas pretenden aprovechar los conocimientos generados para mejorar su competitividad y su rentabilidad.

- OBJETIVO: Incrementar la integración de la actividad investigadora de la UJI con las necesidades industriales del entorno, mediante procedimientos adaptados a los usos empresariales y a las potencialidades y políticas de la Universidad.

ii) Participación en Programas de I+D: La Universidad financia su investigación propia a través de las ayudas que recibe de las diferentes administraciones. Una buena gestión de las ayudas públicas a la I+D, permitirá a los investigadores de la Universidad disponer de una financiación constante y suficiente que garantice su dedicación investigadora. Para ello, es necesario un buen conocimiento de las fuentes de financiación, mantener un contacto permanente con éstas y difundir la información entre la comunidad investigadora. Así mismo, es necesario ayudar al investigador a preparar y tramitar sus propuestas, incrementando la homogeneidad y calidad de las mismas en el conjunto de la Universidad.

- OBJETIVO: Incrementar la cantidad de financiación pública para I+D que recibe la Universidad, a través de buenas prácticas en la gestión de la información y en la preparación y trámite de las propuestas.

iii) Difusión y transferencia de tecnología: La difusión y transferencia de tecnología comprende un conjunto de tareas encaminadas a situar en el mercado los resultados de la investigación universitaria que tengan aplicación industrial. Con ello, se puede extraer rentabilidad social a las inversiones en 
formación de investigadores y proyectos de investigación en el ámbito universitario. La Universidad, a través de este proceso obtiene no sólo beneficios económicos, sino también prestigio social.

- OBJETIVO: La Universidad Jaume I pretende a través de este proceso, la comercialización de la tecnología producida en los diferentes departamentos como producto de la actividad investigadora, con el fin de rentabilizar económica y socialmente sus esfuerzos en investigación y desarrollo tecnológico.

iv) Evaluación de la Investigación: La Universitat Jaume I se ha sumado a la iniciativa del Consejo de Universidades de someterse a un proceso de evaluación continua de sus funciones: docente, investigadora y de gestión. Así mismo la Universidad evalúa su rendimiento investigador a través del análisis de su producción científica.

- OBJETIVO: Se pretende la mejora continuada de la función investigadora y aprovechar los datos e indicadores generados como instrumento en el establecimiento de políticas de impulso a determinadas actuaciones.

\section{ESTRATEGIAS}

\subsection{Estrategias básicas}

\subsubsection{ENFOQUE INTEGRADO DE LA GESTIÓN}

La integración de la gestión de los procesos de investigación académica, investigación bajo contrato, transferencia de tecnología y evaluación permite:

i) Una mejor comprensión de los procesos y la sinergia entre ellos.

ii) Apoyo orientado a la estrategia y vocación de cada grupo de investigación

iii) Permite la valorización y comercialización de los resultados

iv) Es adecuado a una universidad pequeña

\subsubsection{COORDINACIÓN CON OTROS SERVICIOS DE LA UNIVERSIDAD}

La OCIT está integrada en la universidad como un servicio de gestión más. Para el desarrollo de sus funciones trabaja coordinadamente con otros servicios universitarios, tales como el Servicio de Gestión Presupuestaria, el Servicio de Intervención y Auditoría Contable o el Servicio de Recursos Humanos.

Por otra parte la OCIT centraliza todos los contratos de I+D que suscribe la Universitat Jaume I, para ello se relaciona con las otras dos unidades de interfaz universidad-empresa existentes: la Fundación Universitat Jaume I - Empresa y el Instituto de Tecnología Cerámica. De esta manera la OCIT realiza la defensa de los intereses de la Universidad y posibilita la homogeneización de criterios en el establecimiento de relaciones contractuales.

\subsubsection{SISTEMA DE INFORMACIÓN}

Uno de los principales retos asumidos por la OCIT ha sido el de dotarse de un sistema de información adecuado al volumen y variedad de gestión que le ha sido confiado. este sistema entró en funcionamiento el 1 de enero de 1999 y recibe el nombre de Sistema de Gestión Global de la Investigación y la Tecnología (GEGANT). Los principios que han guiado su implantación han sido:

i) Enfoque global de la gestión: integración bajo un mismo sistema de información de la investigación académica, la I+D bajo contrato, la transferencia de tecnología y la evaluación de la investigación.

ii) Completitud histórica de los datos y versatilidad en su tratamiento: posibilidad de extraer informes y estadísticas para análisis y posterior toma de decisiones de gobierno de la universidad.

iii) Prestación de servicios de información on-line a la comunidad universitaria.

Actualmente el sistema presta servicio en las áreas de:

i) Investigación académica: gestión de las ayudas a la I+D (proyectos, movilidad de investigadores, becas, otras ayudas...) que permite la catalogación de las propuestas, evaluación de tasas de éxito, obtención de informes anuales, por departamento e investigador, comparación por áreas de conocimiento o áreas tecnológicas...

ii) Investigación bajo contrato: gestión de clientes, informes por sector industrial, datos de contratación y facturación, control de cobros, control de personal contratado y becarios...

iii) Transferencia de Tecnología: catalogación de los grupos de investigación y su oferta tecnológica, catalogación de los resultados de los proyectos, estado de protección legal, clasificación por sectores industriales...

El desarrollo del módulo de evaluación de la producción de literatura científica será objeto de un próximo proyecto que permitirá la integración en el sistema de las reseñas y documentos completos catalogados según criterios bibliográficos y de gestión. Las publicaciones se asignarán al proyecto y grupo de investigación que las haya generado y a los autores proporcionando una valiosa información a los técnicos de la OCIT que desempeñen funciones de evaluación y transferencia de resultados de investigación.

Toda esta información es servida a la comunidad universitaria a través de la web mediante el sistema de apoyo a la administración y servicios corporativos e-UJIer@ (University Enterprise Resource Planning). Este servicio proporciona acceso para la obtención de información corporativa de la Universitat Jaume I y su gestión, tanto a los administradores de la gestión universitaria como a los administrados. A través de él los investigadores pueden conocer el estado de ejecución contable de sus proyectos, el cómputo de horas de dedicación comprometidas en cada uno de ellos, al estado de facturación y cobros de los contratos de $\mathrm{I}+\mathrm{D}$ y al personal permanente y contratado asignado a cada acción.

\subsection{Estrategias en la investigación bajo contrato}

Las tareas que la OCIT desempeña en este área de trabajo son:

i) Promoción y asesoramiento sobre las posibilidades de contratación, tanto a empresas como a profesores

ii) Intermediación en el establecimiento de las relaciones contractuales y en posibles desavenencias o discrepancias en la interpretación del acuerdo

iii) Seguimiento del cumplimiento de los compromisos por las dos partes: empresa e investigadores

iv) Apoyo administrativo para la facturación y cobro

v) Gestión de becas y contratos laborales con cargo a los contratos de $\mathrm{I}+\mathrm{D}$ 
El establecimiento de un marco administrativo claro ha sido fundamental para el relanzamiento de las actividades de investigación bajo contrato y prestación de servicios de carácter técnico. La actual Normativa de Gestión Administrativa de los contratos suscritos al amparo del art. 11 de la LRU, elaborada por la OCIT con la colaboración de otros servicios de la universidad en 1996 ha permitido una gestión más ágil, que con la puesta en marcha del sistema eUJIer@ antes aludido ha ganado en transparencia y prontitud en la información.

La OCIT ha establecido unas bases claras para la contratación, con la preparación de contratos tipo que ayudan al investigador a comprender la naturaleza jurídica de la prestación de su servicio y ayudan al encauzamiento de la negociación con la empresa.

La presencia de la OCIT entre el tejido empresarial se materializa de la siguiente manera:

i) Establecimiento de relaciones y contactos con otras entidades de intermediación o apoyo a las empresas (asociaciones empresariales, Cámara de Comercio, institutos tecnológicos, organismos de la administración...)

ii) Promoción directa a las empresas mediante entrevistas y presentaciones en solitario o conjuntamente con los departamentos universitarios.

iii) Asistencia a ferias

iv) Edición de materiales (catálogos, páginas web...). En este sentido La OCIT dispone de un catálogo que recoge la oferta científico-técnica de los grupos de investigación de la Universidad. Actualmente dicha oferta tecnológica puede ser consultada vía internet en la dirección http: / / sic.uji.es/ocit/grups/.

Con todo ello, y de acuerdo con las directrices que emanan del Plan Estratégico e la Universitat Jaume I, aprobado a principios del año 2000, aún quedan retos por afrontar en este campo:

i) El establecimiento de una política de costes de la investigación que permita la recuperación de los costes totales en los contratos de I+D. A ello contribuirá la implantación del sistema de contabilidad analítica previsto para el año 2001.

ii) Avanzar en la implantación de una visión de la universidad como un socio tecnológico en igualdad de derechos con las empresas, de manera que sea posible aplicar criterios de participación en los resultados más acordes con las aportaciones de la universidad que le permitan recuperar sus inversiones en las acciones de $\mathrm{I}+\mathrm{D}$ de riesgo.

iii) Incrementar el número de grupos de I+D con potencialidad que prestan servicios tecnológicos o de investigación a empresas, como medio de incrementar la financiación y consecuentemente los medios disponibles en los laboratorios.

La evolución de la contratación de I+D y servicios tecnológicos en la Universitat Jaume I se refleja gráficamente a continuación:

EVOLUCIÓN DE LA FINANCIACIÓN DE CONTRATOS DE I+D DESDE LA CREACIÓN DE LA UNIVERSITAT JAUME I Importe de los contratos

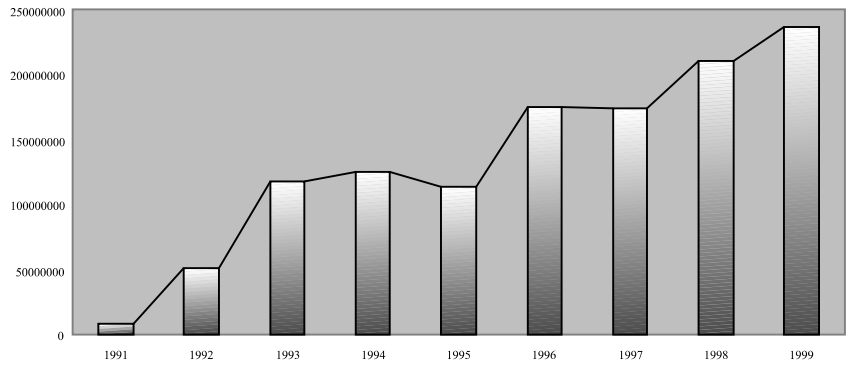

\subsection{Estrategias en la investigación académica}

Las tareas que la OCIT desempeña en este área de trabajo son:

i) Gestión de la información sobre posibilidades de financiación de actividades de I+D

ii) Apoyo en la preparación de propuestas

iii) Trámite de las propuestas ante los organismos financiadores y seguimiento hasta su resolución

iv) Apoyo administrativo y seguimiento de los expedientes concedidos

La OCIT, con la ayuda del Servei d'Informació del Campus (Servicio de información web de la Universitat Jaume I), ha desarrollado un sistema electrónico propio de difusión de las ayudas a la I+D. Dicho sistema consta de una sección en la web y de un boletín electrónico que se emite a una lista de distribución a la que se pueden suscribir los investigadores de la universidad. El sistema recibe el nombre de Flaix $I+D$.

Una estrategia fundamental de la OCIT ha sido reforzar la ayuda y orientación al profesorado a la hora de seleccionar o encauzar sus proyectos o acciones hacia los diferentes instrumentos financieros que se encuentran a su alcance. Actualmente, el sistema de la financiación pública de la I+D es suficientemente complicado como para necesitar el apoyo de servicios especializados, que estudien las diferentes posibilidades e intenten optimizar los esfuerzos de los investigadores en la captación de recursos externos.

Los servicios que provee la OCIT son:

i) Asesoramiento sobre las vías financieras más adecuadas para la acción propuesta

ii) Cálculo del presupuesto de la acción

iii) Consejo sobre la estrategia más adecuada para la propuesta: planteamiento general, necesidad de otros socios...

iv) Consultas previas con las instituciones convocantes con el objeto de calibrar el interés y la adecuación de la propuesta a los objetivos de la convocatoria

v) Búsqueda de socios: universidades, centros públicos de investigación o empresas

La evolución de la financiación de I+D académica desde la creación de la Universitat Jaume I se refleja gráficamente a continuación:

EVOLUCIÓN DE LA FINANCIACIÓN DE PROYECTOS DE I+D DESDE LA CREACIÓN DE LA UNIVERSITAT JAUME I Importe de los proyectos

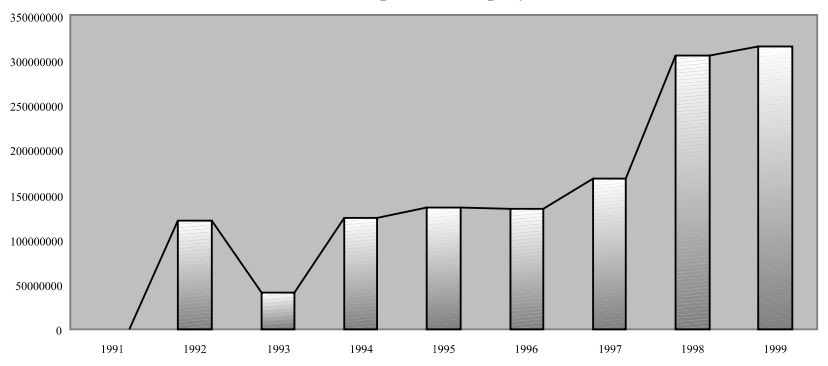

\subsection{Estrategias en la difusión y transferencia de tecnología}

La transferencia de tecnología en el seno de la Universitat Jaume I se lleva a cabo principalmente a través de la investigación bajo contrato. En 1999 el presupuesto de los contratos de investigación orientados a tecnologías cerámicas supuso el $54 \%$ del total de la contratación de I+D de la Universitat 
Jaume I, mientras que este mismo dato referido a la investigación académica apenas alcanza el 5\%. Ello significa que la producción de resultados de interés industrial cuya titularidad sea atribuible a la universidad es y continuará siendo a corto plazo muy limitado, tanto más en cuanto que el principal cliente empresarial de la universidad corresponde a las empresas cerámicas.

A medio plazo, el incremento de la participación de la universidad en proyectos consorciados, requerirá poner a punto mecanismos de protección, valorización y comercialización de resultados, dado que en este tipo de proyectos la propiedad de resultados le es atribuida a la universidad.

\section{LA INVESTIGACIÓN CERÁMICA EN LA UNIVERSITAT JAUME I}

El grueso de la investigación en tecnología cerámica que se desarrolla en la Universitat Jaume I corresponde a desarrollo de fritas, esmaltes y colores o a tecnologías de proceso y de producto acabado. Campos como la investigación y desarrollo de bienes de equipo e instrumentación o cerámicas técnicas han sido escasamente explorados. Ello ha podido ser motivado en parte por una falta de presencia empresarial o de dinamismo de los sectores implicados. Sin embargo los estudios relativos a organización y gestión de la empresa cerámica van cobrando cada vez más peso, con una fuerte presencia en los departamentos de Administración de Empresas y Marketing y de Tecnología.

De las 25 empresas que en la actualidad conforman la Asociación Española de Fabricantes de Fritas, Esmaltes y Colores Cerámicos (ANFFECC), la Universitat Jaume I ha ejecutado investigación bajo contrato para 14, lo cual supone una cobertura del $56 \%$. Ello es especialmente relevante en este subsector, que basa su competitividad en la innovación de producto, y en el que por tanto son habituales fuertes inversiones en $\mathrm{I}+\mathrm{D}$, pero también explica que dichas empresas se rodeen de los socios tecnológicos que consideran que pueden aportarles el conocimiento y los medios humanos y materiales que precisan. Cabe además citar que en la mayor parte de los casos la colaboración reviste carácter de regularidad, con lo cual puede hablarse de una verdadera cooperación tecnológica, en la que se busca la satisfacción de los intereses mutuos y el respeto y la comprensión de las limitaciones de cada socio.

Sin embargo la cobertura en otros sectores es más reducida: las empresas fabricantes de baldosas y azulejos que han contratado $\mathrm{I}+\mathrm{D}$ a la Universitat Jaume I coinciden con lo que se ha dado en llamar "líderes tecnológicos". En este sentido, aunque el número de empresas que se han acercado a la universidad es escaso, cabe decir que se ha desarrollado investigación bajo contrato para la práctica totalidad de empresas que destacan por sus procesos de innovación y por asumir riesgos tecnológicos.
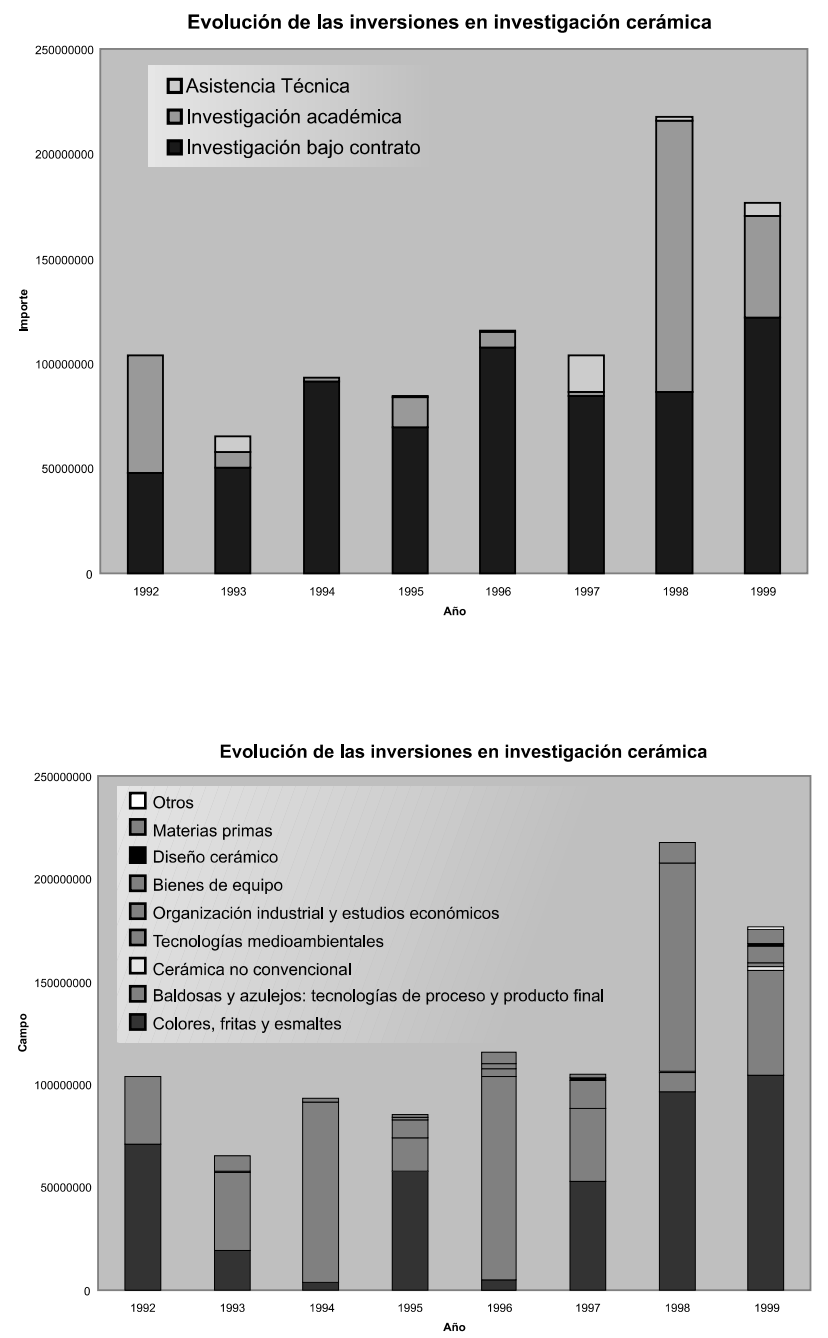

Es de destacar la escasa actividad investigadora en instrumentación y bienes de equipo, aunque es de esperar que la interacción entre las empresas del sector y departamentos de la universidad como el de Tecnología o el de Informática se incremente a medio plazo. En este sentido ya se han realizado contactos entre la asociación del sector, ASEBEC, y la universidad con el objetivo de dinamizar la relación.

\section{NOTAS}

*Memoria de la Propuesta del Proyecto "Estudio del impacto regional de la Titulación de Licenciatura en Ciencias Químicas de la Universitat Jaume I de Castellón". Documento interno del Vicerrectorado de Investigación y Desarrollo. 